\title{
Design approach for the development of a digital twin of a generic hybrid lightweight structure
}

\author{
Tobias Ryl1 ${ }^{*}$, Moritz Kuhtz ${ }^{1}$, Lukas Quirin ${ }^{1}$, Minh Nguyen $^{1}$, Maik Gude $^{1}$ and Angelos \\ Filippatos ${ }^{1,2}$ \\ ${ }^{1}$ Institute of Lightweight Engineering and Polymer Technology (ILK), \\ Technische Universität Dresden, Holbeinstr. 3, 01307 Dresden, Germany \\ ${ }^{2}$ Dresden Center for Intelligent Materials (DCIM), \\ Technische Universität Dresden, 01069 Dresden, Germany
}

\begin{abstract}
The innovation potential of lightweight design in its scientific and technical foundations lies in the systematic, i.e. integrated and crossmaterial consideration of the process chains. This consideration is one of the main goals of dahlia project - Digital Technologies for Hybrid Lightweight Structures. The end-to-end digitalisation of the development, manufacturing and validation processes provides a first understanding of its process parameter-structure-property relationships, which is essential for developing a representative digital twin. To investigate these aspects in the design phase profiles made of aluminium alloy are exemplarily studied. Important aspects for the development of a digital twin are considered, such as a knowledgebased selection of design and simulation tools, their respective parametrisation and interface communication. First results are presented as well as the development aspects of a digital twin from its nominal offline form up to its specific application at a generic lightweight structure.
\end{abstract}

\section{Introduction}

With the need for increasingly lighter components the industry is driven to more complex hybrid materials. Nevertheless, hybrid structures are rarely applied in the industry. Especially in SMEs in Europe the use is still not common due to the high investment cost and challenges with the digital infrastructure.

New material combination like lightweight metal sheets reinforced with fibre reinforced thermoplastic (FRP) making the development process more complex [1]. The mechanical properties of hybrids are not previously fully determined, that means most properties are created during the manufacturing of the metal and the composite. Forced due to the previous, it can be considered, that also the design approach has to be further adjusted to fit the current industry needs. To decipher the complexity of the production cycle means to understand correlations and material behaviours of the process chain starting by the manufacturing of the materials and ending by the surveillance of the produced components [2]. One contribute to this is the end-to-end digitalisation of the development, manufacturing and validation processes of hybrid lightweight structures that enables a profound understanding of their

* Corresponding author: Tobias.Ryll@tu-dresden.de 
process parameter-structure-property relationships (PSP relationships). Established design approaches such as VDI-2221 or the QFD approach must be improved and extended to meet these new requirements. Technologies to aid the networking of manufacturing processes and combine them with corresponding simulation and other software tools, have already been investigated [3]. A suitable tool that can be added into this are digital twins (DT) [4, 5]. A DT is a concept in which a physical system is represented by a virtual counterpart with specific functionalities and covering all life phases. With regard to Industry 4.0, DTs represent one of the key technologies for online structure monitoring $[4,6]$.

One of the goals of the dahlia project (Digital Technologies for Hybrid Lightweight Structures) is to provide the industry with the needed design approach, so that hybrid materials establish themselves in the industry. The most important innovation goal of the project is the systemic, i.e. continuous and cross-material consideration of the entire process chain. This design approach for hybrid structures aims at disciplinary networking of the individual activities and thus makes it possible to decode the PSP relationships by means of simulation and to prepare them for the use in DTs [7]. This is a core element of industry 4.0 and accelerates the trend towards decentralisation of manufacturing and production $[8,9]$.

Until now, the production of load-adapted multi-layer structures made of light metal sheets in combination with thermoplastic FRP has been developed without problem-adapted simulation and design tools. Currently, the technologies and approaches regarding a DT of a hybrid material structure are complex and expensive. This prevents their use in the industry. The knowledge gained with the new approach is used to derive an application-specific, webbased simulation and design tool that can be operated without special expertise making the use less complex and cheaper. Figure 1 shows the methodology of the development process in the dahlia project.

In this article, an approach for the development of a part of a DT for a hybrid structure is shown by means of a digital shadow (DS) of a demonstrator. In this context, a DS is a variant of a DT that is limited in terms of data streams or open-loop and closed-loop control capabilities [5]. For the demonstration purposes, a small sized bench was manufactured and equipped with distance sensor technology. The data received from the sensor was processed by a Raspberry Pi and sent to a server. A web-based and flow-based programming tool, such as Node-Red developed by the company JS Foundation, can be used to access this data and monitor the demonstrator from any location with an Internet connection.

\section{State of the Art}

Hybrid materials are materials consisting of two dissimilar components joined as a single entity e.g. magnesium sheets reinforced with FRP [10]. Their properties can be either enhanced or completely new. Hybrid materials can be found for all kind of applications in technology, in everyday use products and in nature [11]. Although hybrid material structures are already state of the arts the development with analogue process methods is often inefficient. Especially the simulation of this material's behaviour with analogue process methods is still accompanied by vast difficulties due to the enormous number of possible material property parameters and their complex interactions [12].

Moreover, process influences on parts e.g. thermally induced residual stresses or distortions lead to challenges in the prediction of the structural behaviour. Further challenges lie in the forming processes, the evaluation of the model, and the access to monitor the manufacturing quality. In addition, there are already challenges in the development of composite materials that further increase complexity. 


\subsection{Methods and Materials}

In the presented study, magnesium profiles with tape reinforcements are used as an example showing the need for the new design approach. In this approach non-desirable phenomena such as ghost lines in the magnesium sheets, deficient adhesiveness between the tapes and the profiles or problems that only occur due to combination of parameters but not because of a single parameter can be studied.

The profile analysed during the dahlia project is a tape-reinforced light metal profile. Non-isotropic magnesium alloy profiles are reinforced with unidirectional glass fibre reinforced thermoplastic tape layers. In order to represent the complexity of the material property interactions, the entire manufacturing chain is considered, i.e. the magnesium sheet and tape production, the roll producing of the profiles and the application process of the tapes. The glass fibre reinforced PA6 thermoplastic tapes are produced in a production line where the most important manufacturing parameters such as temperature, sizing quality, yarn tension and yarn velocity are monitored. The magnesium alloy (ZAX210) is manufactured in a pilot plant. Due to the properties of the alloy, the magnesium sheets can be bent at room temperature [13]. The profile shape is then formed by roll forming. During calibration rolling, the tapes are applied to the profiles in an inline laying process.

For the dahlia project, the individual manufacturing processes of the materials are distributed among the three project partners involved, which also ensures a spatial separation of data acquisition and data processing. Two institutes contribute the production including the acquisition of parameter data. The third institute, the Institute of Lightweight Material and Polymer Technology at the TU Dresden (TUD-ILK), provides the process simulation and data architecture for the DT technology based on the collected manufacturing parameter data, shown in Figure 1.

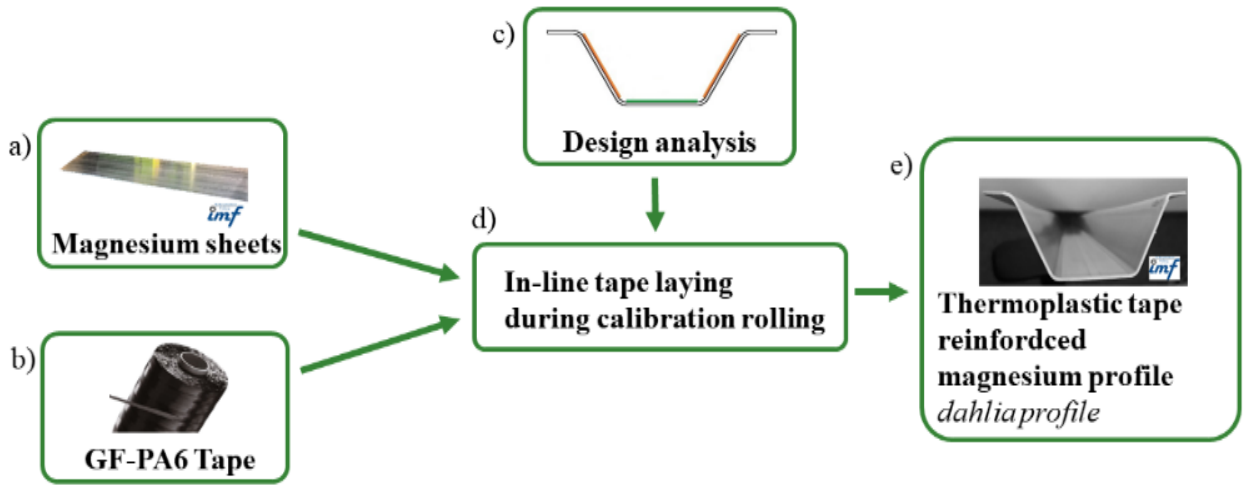

Fig. 1 Concept of the produced profile., with a) Magnesium sheet manufacturing , b) GF-PA6 Tape manufacturing c) Design, simulation and material model providence d) In-line tape laying of a GFPA6 tape during roll forming of a magnesium sheet e) End product: manufactured thermoplastic tape reinforced magnesium profile

\subsection{Digital twin concept for hybrid structures}

Understanding the advantages of material combinations and challenges arising from a wide range of possible parameters is crucial for simulating and determining the process-parameter-structure-property relationships [3,9]. The new approach is to develop and use a DT to help the designing process of a component and to understand the PSPrelations. The application of the method takes into account a demonstrator and additionally a hybrid material system including the manufacturing process chain with its DT and its hybrid model respectively. The basic concept approach for a universally applicable DT is shown in 
Figure 2. The main parts are middleware, such as the application programming interface (API), the backend, the frontend and input data from the user and different edge-components. The edge-components can be hardware components, such as workstations that collect data from machines.

Middleware forms the basis of the system and describes a toolset used in many IT applications where data has to be exchanged between various components of a system or process respectively. It consists of the central API and all other software interfaces, services and protocols that are necessary to ensure communication from the edge devices to the backend, as well as from the backend to the frontend [14]. There's already multiple protocol standards available. The selection of the appropriate protocol is based on, for example, the amount of data, hardware and users, as well as the type of communication and reliability [15]. The API controls access and communication between all components and applications of the DT infrastructure.

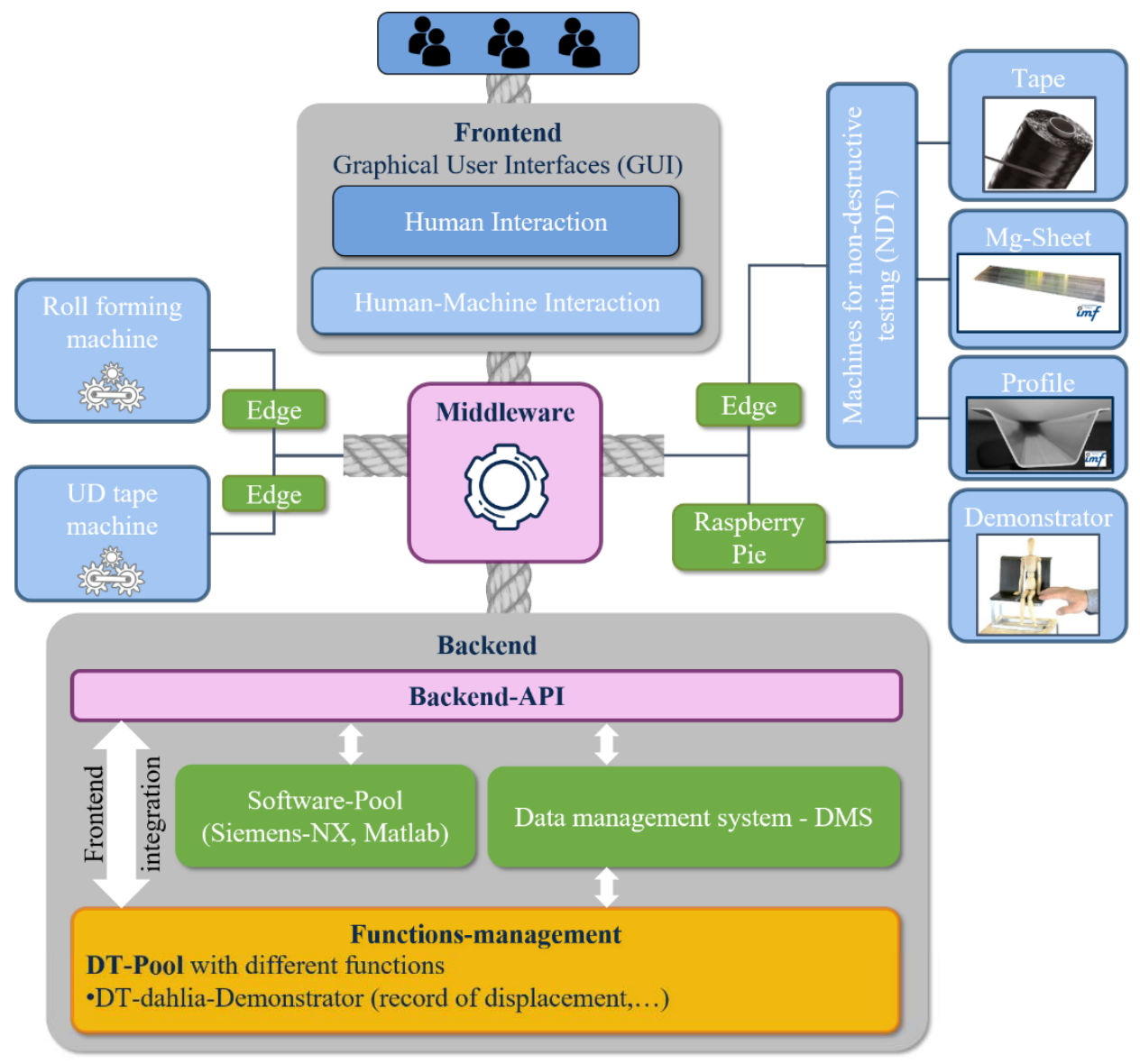

Fig. 2 Digital twin - Basic concept of the digital structure of the Dahlia project with its components, the backend, the frontend, various edge devices and user inputs that transmit data via the central component, the middleware.

The frontend is the interface between the API and the user. It contains various general user interfaces (GUI) such as web apps or machine interfaces. It allows the user to visualise and manipulate data collected from sensors for instance, to perform calculations such as averaging, standard deviations, etc. the backend is used to permanently store data and make 
it available for further calculations. It can also independently perform online calculations using models. These models are derived from simulations or by other evaluation methods. They enable the DT to perform correlations and thus predictions about the current or even future performance of the structure. In addition, the backend contains meta data such as information about how the data is stored and who has access rights to it. It is therefore also used for administrative and organisational tasks (e.g. licensing, data management, function management, etc.).

\section{Application on a concept demonstrator}

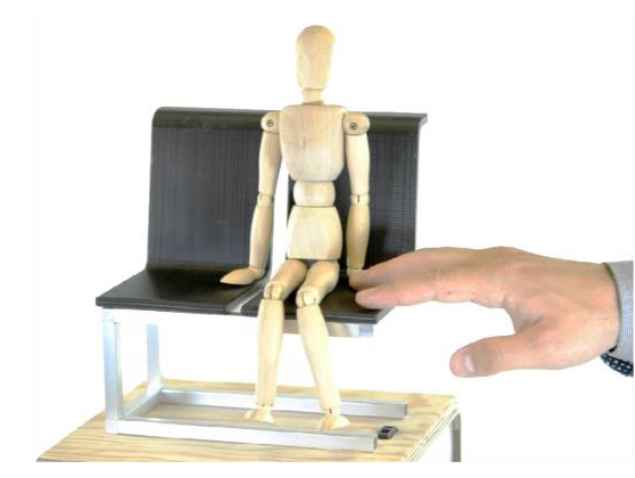

a)

Fig. 3. Table-sized demonstrator (a), dimensions of the profile (b) and simplified simulation model with dimensions (c).

The approach shown in this paper is applied to a simplified and table-sized demonstrator. Figure 3 shows the dimensions and an image of the demonstrator next to a hand. The bench is build up out of aluminium profiles of the dimensions $10 \mathrm{~mm} \times 10 \mathrm{~mm}$ with a thickness of $1 \mathrm{~mm}$. Aluminium was chosen because it was easier and quicker to procure. This demonstrator is intended to illustrate the principle functioning of our DT concept and its key characteristics are noted in table $\mathbf{1}$.

Table 1. Key characteristics of the demonstrator

\begin{tabular}{lcc}
\hline Description & Value & Unit \\
\hline Dimensions & $85 \times 205 \times 85$ & $\mathrm{~mm}$ \\
Force & 10 & $\mathrm{~N}$ \\
Sensor Sampling Rate & 4 & $\mathrm{~Hz}$ \\
Data Transmission Rate & 1 & $\mathrm{~Hz}$ \\
Displacement & 5 & $\mathrm{~mm}$ \\
Input Power & 15 & Watts \\
\hline
\end{tabular}

*The values represent maximum values and may vary during operation.

The complex hybrid material behaviour described above is not yet to be investigated at this point. To understand the stress distribution a linear implicit finite element analyses was created with Siemens NX. The aluminium profiles as the load-bearing structure were modelled using 1D beam elements and a linear elastic isotropic material model. The connections of the profiles were assumed as ideally stiff and hence realised by means of 
merged nodes. In the experiment, the outer part of the demonstrator is pushed downwards to simulate a person sitting on the bench. In the simulation model, as boundary conditions, the elements of the lower profiles are fixed in all dimensions and additionally a time variable load is applied on the two outer nodes to map the actual load case. A sketch of the model with its load and boundary conditions is shown in Figure $3 \mathrm{c}$ ). The simplified digital architecture of the demonstrators DT is shown in Figure 4. An infrared distance sensor continuously measures the distance between the bottom and the seat and converts it into a

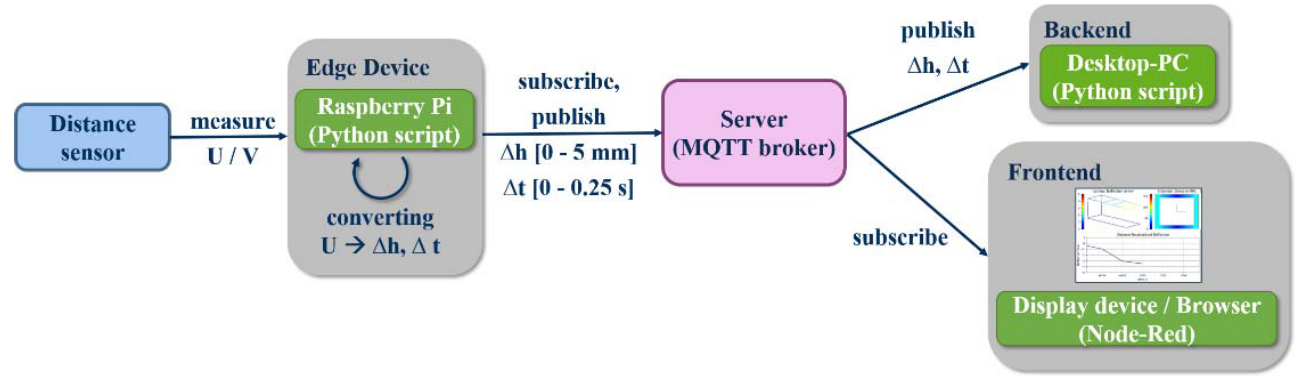

Fig. 4 Data workflow of the table-sized demonstrator from edge device to its frontend and backend respectively.

displacement $\Delta h$. The Edge device, which is a Raspberry Pi $4 \mathrm{~B}$, retrieves time-discrete measurement data. Out of this data, the input for the frontend is distributed via a Message Queuing Telemetry Transport protocol (MQTT protocol) serving as the middleware broadcasting data to any other device. Since the table-sized demonstrator is a minimalist initial design and only small amounts of data need to be transmitted, the relatively easy-toimplement MQTT protocol was used. The data processing can be done with a desktop-PC and the visualisation can be done at any display device in a browser. In this example, the desktop-PC serves as backend due to its storing, computing and visualising capabilities. The calculations and visualisation are performed via a Python script.

On any frontend device, e.g. a handheld device, the data visualisation of the DT can be performed live with a web-based application such as solutions from Node-RED or Apache Zeppelin. A first example of data visualisation can be seen in Figure 5, where a model and cross-section with corresponding stresses can be found at the top and the data progression is
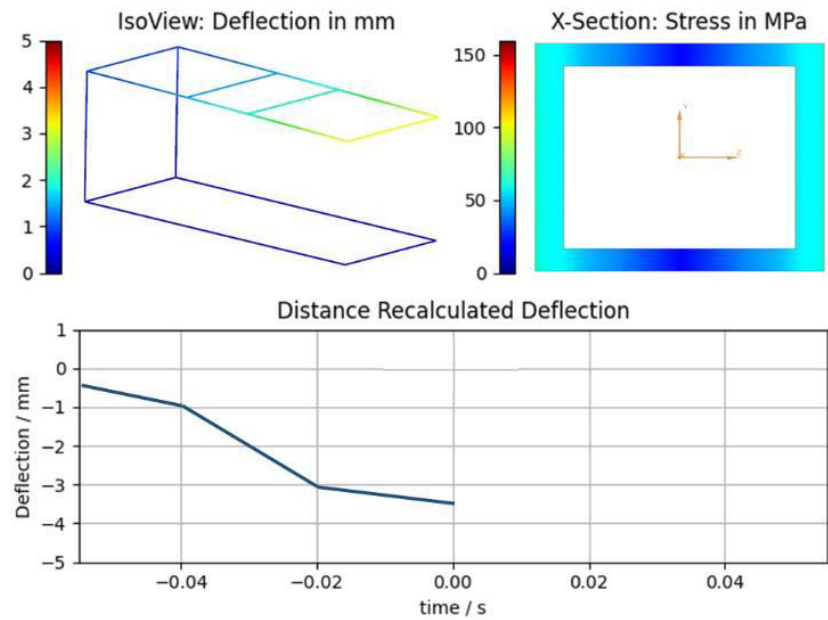

Fig. 5 Screen capture of the frontend of the table sized demonstrator. 
shown in a standard plot at the bottom. In the experiment, the distance measurements are converted into displacements, which are then compared to deflection stress patterns of the bench previously computed in simulations. It can be seen that the main purpose of the frontend is pure visualisation and user-machine interaction. Any calculations are mainly performed by the backend. However, minor calculations such as the conversions from distance to displacement can be performed directly by the edge device.

\section{Discussion and Outlook}

The approach presented is the first attempt to demonstrate the potential of DT technology and the complexity that arises when this technology is used on a hybrid lightweight structure. The method to develop a DT was successfully applied on a first state DS onto a table-sized demonstrator. The data being part of the DS is stored on a server and can be accessed from everywhere with an internet connection. Furthermore the live state of the demonstrator can be retrieved and processed. The visualisation of the live state and the data history was implemented and can be monitored from anywhere else as well.

After the first demonstrator being designed in table size out of aluminium profiles, the next step is to use this approach on a full sized hybrid material based demonstrator that can be used in public places or vehicles, e.g. public transport, air planes etc. With this, more difficulties such as the complex material behaviour and the scalability of the approach with more sensors can be analysed. Additionally, the manufacturing history of individual parts of a demonstrator should be added to rise the complexity and accuracy of the DS towards becoming a DT. Concluding, the first results of a DS of an aluminium alloy demonstrator bench are presented and show the potential of a systematic lightweight design approach. A simplified demonstrator was successfully implemented as a first step towards a DT of a hybrid cross-material out of a newly developed magnesium alloy and fibre reinforced plastic. Parts of the approach of considering the whole process chain of a cross material and creating a digital copy were presented. This first step shows that further work has to be done for developing a complete DT of a hybrid lightweight structure to investigate a full DT of a hybrid out of a fibre reinforced plastic and a metal.

\section{Declaration of Competing Interest}

The authors declare that they have no known competing financial interests or personal relationships that could have appeared to influence the work reported in this paper.

\section{Acknowledgement}

The authors gratefully acknowledge the financial support for this research provided by the European Union (European Regional Development Fund) and the Free State of Saxony under grant agreement no. 100383375 ("dahlia"). We thank for the financial funding of the Dresden Centre for Intelligent Materials (DCIM) by the Free State of Saxony and TU Dresden.

\section{References}

1. M. Irani, M. Kuhtz, M. Zapf, M. Ullmann, A. Hornig, M. Gude, U. Prahl: Investigation of the Deformation Behaviour and Resulting Ply Thicknesses of Multilayered FibreMetal Laminates. J. Compos. Sci. 5, 176 (2021)

2. M. Mozaffar, A. Paul, R. Al-Bahrani, S. Wolff, A. Choudhary, A. Agrawal, K. Ehman, J. Cao: Data-driven prediction of the high-dimensional thermal history in directed energy deposition processes via recurrent neural networks, in Manufacturing Letters, 18, 35-39 (2018) 
3. J. Wollmann, D.R. Haider, M. Krahl, A. Langkamp, M. Gude: Linked a Priori and a Posteriori Models of Composite Manufacturing Process Chain. E. Rusiński, D. Pietrusiak (eds) Proceedings of the 14th International Scientific Conference: Computer Aided Engineering. CAE 2018. Lecture Notes in Mechanical Engineering. Springer, Cham (2019)

4. B.R. Barricelli, E. Casiraghi, D. Fogli: A Survey on Digital Twin: Definitions, Characteristics, Applications, and Design Implications. IEEE Access. 7 (2019)

5. W. Kritzinger, M. Karner, G. Traar, J. Henjes, W. Sihn: Digital Twin in manufacturing: A categorical literature review and classification. IFACPapersOnLine. 51, 1016-1022 (2018)

6. S. Haag, R. Anderl: Digital twin - Proof of concept, in Manufacturing Letters, 15, Part B, 64-66 (2018)

7. Filippatos, Angelos, Albert Langkamp, and Maik Gude. Influence of gradual damage on the structural dynamic behaviour of composite rotors: Simulation assessment. Materials, 11, 2453 (2018)

8. E. Negri, L. Fumagalli, M. Macchi: A review of the roles of Digital Twin in CPS-based production systems. Procedia Manufacturing, 11, 939-948 (2017),

9. F. Psarommatis: A generic methodology and a digital twin for zero defect manufacturing (ZDM) performance mapping towards design for ZDM, in Journal of Manufacturing Systems, 59, 507-521 (2021)

10. Z. Huang, S. Sugiyama, J.Yanagimoto: Adhesive-embossing Hybrid Joining Process to Fiber-reinforced Thermosetting Plastic and Metallic Thin Sheets, in Procedia Engineering, 81, 2123-2128 (2014)

11. G.L. Drisko, C. Sanchez: Hybridization in Materials Science - Evolution, Current State, and Future Aspirations. European Journal of Inorganic Chemistry (2012)

12. M. Kuhtz, N. Buschner, T. Henseler, A. Hornig, M. Klaerner, M. Ullmann, H. Jäger, L. Kroll, R. Kawalla: An experimental study on the bending response of multi-layered fibre-metal-laminates. Journal of Composite Materials, 53, issue: 18, 2579-2591 (2019)

13. M. Ullmann, K. Kittner, T. Henseler, A. Stöcker, U. Prahl, R. Kawalla: Development of new alloy systems and innovative processing technologies for the production of magnesium flat products with excellent property profile, in Procedia Manufacturing, 27, p. 203-208 (2019)

14. M. A. A. da Cruz, J. J. P. C. Rodrigues, J. Al-Muhtadi, V. V. Korotaev, V. H. C. de Albuquerque: A Reference Model for Internet of Things Middleware, in IEEE Internet of Things Journal, 5, no. 2, 871-883 (2018)

15. A. Eckhardt, S. Müller, L. Leurs: An Evaluation of the Applicability of OPC UA Publish Subscribe on Factory Automation use Cases, in Proceedings of the 2018 IEEE 23rd International Conference on Emerging Technologies and Factory Automation, ETFA, 1071-1074 (2018) 